\title{
Clinics in diagnostic imaging (172)
}

Hsien Min Low $^{1}$, MBBS, FRCR, Dinesh Chinchure $^{2}$, MBBS, FRCR

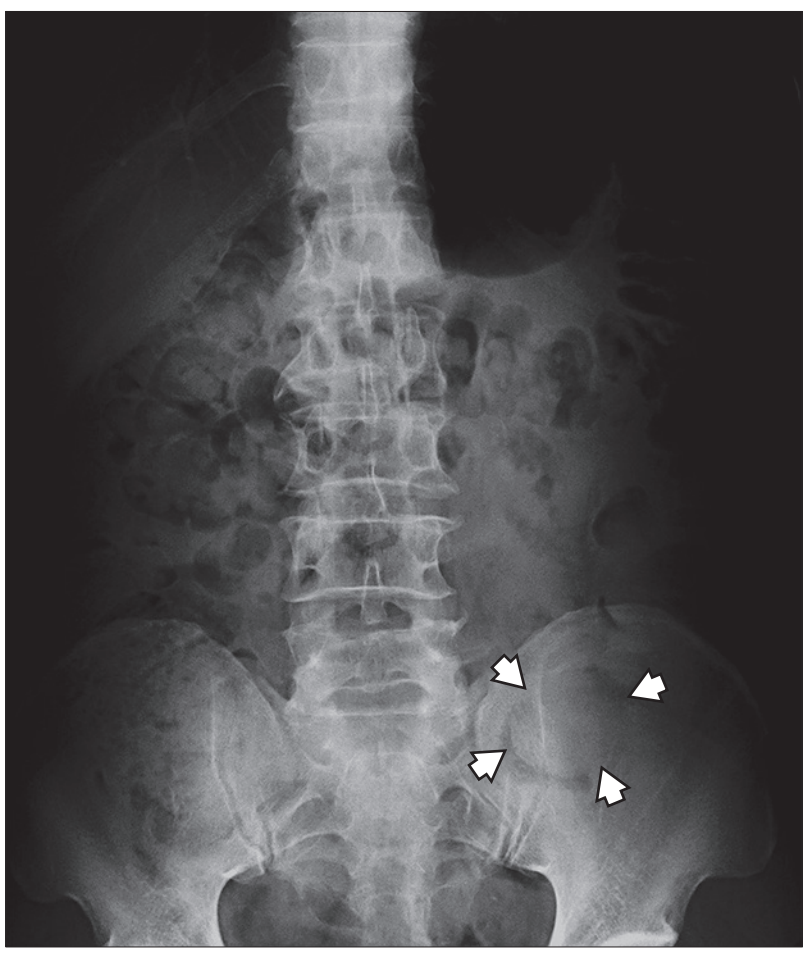

Fig. 1 Supine radiograph of the abdomen.
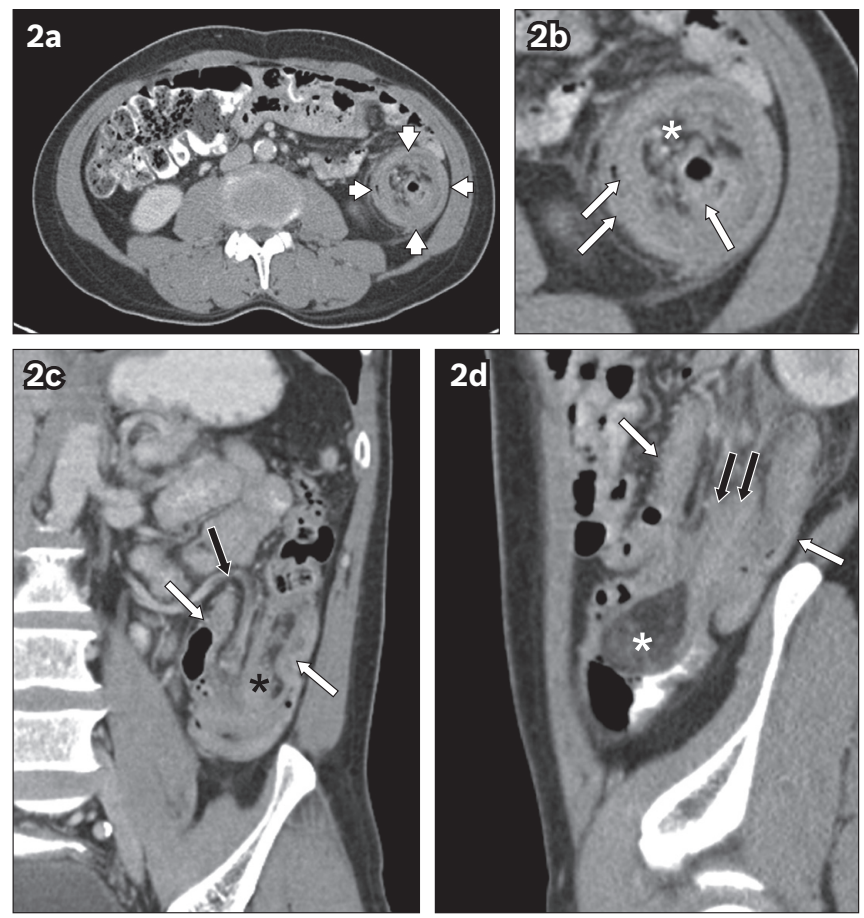

2d

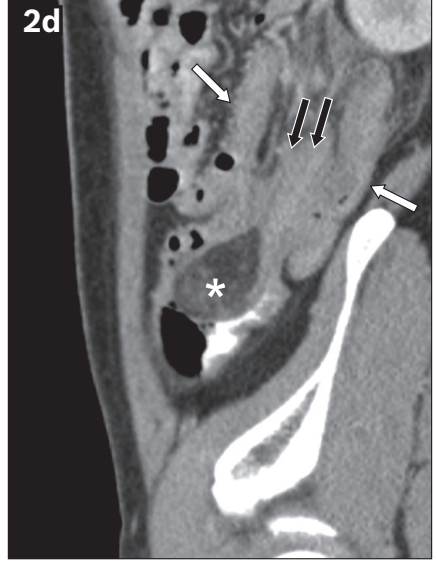

Fig. 2 (a \& b) Axial and magnified; (c) coronal; and (d) sagittal CT images of the abdomen and pelvis in the portal venous phase.

\section{CASE PRESENTATION}

A 50-year-old Chinese man who had no significant past medical history presented with a one-month history of bloody stools mixed with mucus. This was associated with suprapubic abdominal pain, loss of appetite and weight loss of $4 \mathrm{~kg}$ over the past month. He did not complain of haematemesis, chest tightness or giddiness.
Physical examination revealed mild suprapubic tenderness with no evidence of guarding or rebound tenderness. The bowel sounds were active and digital rectal examination showed soft brown stools within the rectum, with no melaena or mass felt. Supine abdominal radiography (Fig. 1) and contrast-enhanced computed tomography (CT) of the abdomen and pelvis (Fig. 2) were performed. What do these images show? What is the diagnosis? 


\section{IMAGE INTERPRETATION}

The supine abdominal radiograph (Fig. 1) shows an ovoid opacity in the left lower quadrant that appeared to be outlined by gas (arrowheads). No intestinal obstruction or pneumoperitoneum is seen. On the axial CT image (Fig. 2a), a targetoid lesion is seen in the region of the left flank (arrowheads), which corresponds to the opacity noted on the abdominal radiograph. Magnified view of the lesion (Fig. 2b) shows a 'bowel-within-bowel' appearance with three concentric, alternating layers of soft-tissue density bowel walls (arrows) and low-density mesenteric fat (asterisk). Coronal CT image (Fig. 2c) demonstrates the proximal segment of the descending colon (asterisk) prolapsing into the distal segment (white arrows). The mesenteric vessels and accompanying fat also appear to be dragged along with the herniated bowel loop (black arrow). The sagittal image (Fig. 2d) shows an ovoid low-density mass measuring $4.1 \mathrm{~cm} \times 2.2 \mathrm{~cm}$ at the apex of the invaginated bowel (asterisk). Herniation of the proximal descending colon (black arrows) into the distal segment (white arrows) is re-demonstrated.

\section{DIAGNOSIS}

Colocolic intussusception with a lipoma as the lead point.

\section{CLINICAL COURSE}

On-table sigmoidoscopy performed on the patient showed a polypoid whitish mass $30 \mathrm{~cm}$ from the anal verge, which was completely occluding the lumen. He subsequently underwent a laparotomy that showed no evidence of a colocolic intussusception. However, the distal segment of the sigmoid colon was noted to be oedematous and indurated. A hard, mobile mass was palpable within the descending colon, with no invasion of the surrounding structures. No lymphadenopathy, peritoneal disease or dilated small bowel loops were seen. A left hemicolectomy was then performed. Although there was no intussusception seen during surgery, the imaging findings were unequivocal for intussusception. It can be postulated that the prolapsed bowel spontaneously reduced shortly after the sigmoidoscopy was performed.

Gross pathological examination revealed a polypoid mass measuring $6.6 \mathrm{~cm} \times 3.6 \mathrm{~cm} \times 2.3 \mathrm{~cm}$ in the descending colon, with surface ulceration and haemorrhage. The segment of the colon distal to the mass was oedematous. Histological examination showed the mass within the descending colon to be a submucosal lipoma. There was no evidence of dysplasia or malignancy. The patient's post-surgery recovery was uneventful, with no further complaints on follow-up.

\section{DISCUSSION}

Intussusception is a recognised but rare cause of intestinal obstruction in the adult population. Adult intussusception (AI) makes up $5 \%$ of all cases of intussusception and only $1 \%-5 \%$ of intestinal obstruction in adults. ${ }^{(1)}$ This is in contrast to the paediatric population, in which the most common cause of intestinal obstruction in infants aged 6-36 months is intussusception. $^{(2)}$

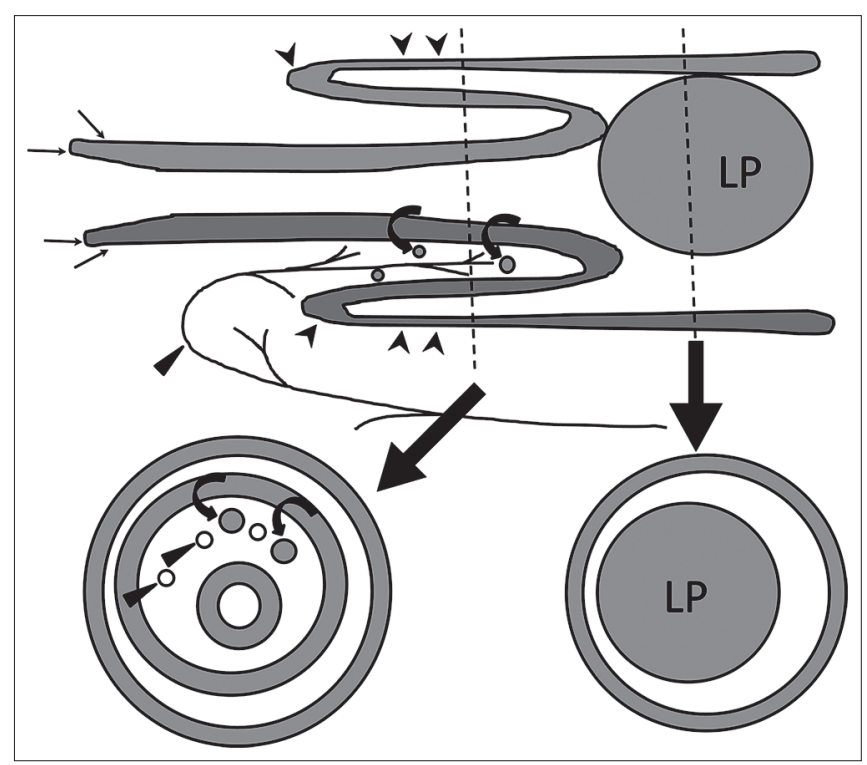

Fig. 3 Schematic diagram of an intussusception shows the telescoping of one segment of the bowel (intussusceptum, thin black arrows) into the adjacent segment (intussuscipiens, black arrowheads). Cross-sectional diagram of the mid-segment of the intussusception shows the classical target-like appearance with three layers of bowel. Note the dragging of the mesenteric vessels (indicated by black triangles) and hyperplastic lymph nodes (curved black arrows) along with the intussusceptum [adapted from Choi SH, et al]. ${ }^{(3)}$ LP: lead point

The condition is characterised by the invagination of a proximal loop of bowel (termed the intussusceptum) into a distal loop of bowel (known as the intussuscipiens), similar to how the parts of a collapsible telescope slide into one another. This gives rise to a 'bowel-within-bowel' appearance that can be recognised on imaging studies. The process of invagination pulls the mesenteric fat and vessels into the plane between the intussusceptum and intussuscipiens. This causes kinking of the vessels, resulting in vascular congestion, bowel ischaemia and perforation in severe scenarios. Fig. 3 is a schematic diagram that shows the longitudinal and serial cross-sectional views of an intussusception. ${ }^{(3)}$

Patients with Al commonly present with nonspecific abdominal symptoms and features of intestinal obstruction that may have an acute, intermittent or chronic course. Only 9\%-10\% of adult patients present with the classic triad: acute colicky abdominal pain; passing of 'currant jelly' stool; and a palpable mass in the abdomen. ${ }^{(4)}$ The location of Als can be broadly divided into enteric and colonic. In a literature review conducted by Felix et al, out of 1,214 adult patients with colonic and small bowel intussusception, 55\% occurred in the small bowel and $45 \%$ were colonic. ${ }^{(5)} 70 \%-90 \%$ of Als have an underlying lesion that acts as a lead point for telescoping to occur. ${ }^{(1)}$ Lead points are thought to either alter the normal peristaltic movements of the bowel, or act as an intraluminal mass that is propelled in the antegrade direction by peristalsis, thereby pulling a segment of bowel along with it.

Numerous entities can act as a lead point, but a malignant tumour of the bowel is the cause for most concern. In Felix et al's literature review, $48 \%$ of colonic intussusceptions (261/546) were found to be due to a malignant tumour; conversely, only 
$17 \%$ of all small bowel intussusceptions (116/668) were due to malignancy. ${ }^{(5)}$ Given that a significant proportion of colonic intussusception in adults is due to malignancy, it is prudent for radiologists to be watchful for radiological features of malignancy, such as lymphadenopathy, irregular polypoid mass and liver metastases. Fig. 4 demonstrates a colorectal intussusception in an adult patient who had a bulky polypoid mass at the apex of the intussusceptum. Histology showed that the mass was an adenocarcinoma.

Small bowel intussusception, a condition seen in both adult and paediatric populations, can be transient and selflimiting. In such cases of transient small bowel intussusception, no lead point can be identified; the length and diameter of the intussusception are shorter and smaller, respectively, and the proximal bowel loops are not dilated. Patients are usually asymptomatic, and telescoping of small bowel loops is occasionally seen when patients undergo CT or ultrasonography for an unrelated condition. ${ }^{(6,7)}$ Fig. 5 shows an incidental small bowel intussusception in a patient who underwent CT of the abdomen and pelvis for evaluation of weight loss and anaemia.

Although a significant proportion of Als have a malignant tumour as a lead point, benign tumours such as lipomas can also be responsible, as shown in our case. Lipomas are the second most common benign tumour of the colon, with an incidence of up to $4 \%$ in autopsy series. ${ }^{(8)}$ They are often discovered incidentally when a patient undergoes a radiological examination for an unrelated cause. Fig. 6 demonstrates a lipoma in the ascending colon that was incidentally detected.

Lipomas are the most common benign tumour found in cases of large bowel AI. ${ }^{(9)}$ From 2001 to 2011, 50 cases of Al induced by a lipoma were reported in the English literature, of which 23 cases were lipomas arising from the colon. ${ }^{(10)}$ The imaging findings of another patient who presented to our institution with intussusception due to a lipoma are shown in Fig. 7. CT showed that a loop of the bowel had prolapsed into the ascending colon and the lipoma can be seen at the apex of the intussusceptum. Surgery performed for this patient revealed a caeco-colic intussusception secondary to a lipoma.

Radiology remains crucial in the diagnosis of intussusception. Plain radiography is helpful to exclude pneumoperitoneum, which would necessitate urgent surgical intervention. In some patients, the intussusceptum may be seen as a soft-tissue mass in the right upper quadrant, outlined by a crescent of gas within the colonic lumen (also known as the 'meniscus sign').

In recent years, multidetector $\mathrm{CT}$ of the abdomen and pelvis has increasingly become the imaging modality of choice for evaluating the adult patient suspected to have intussusception. ${ }^{(9)}$ CT is able to diagnose intussusception by demonstrating a targetlike mass with alternating layers of low-density mesenteric fat and soft-tissue density bowel walls. Enhancing mesenteric vessels are also seen within the mesenteric fat as it accompanies the intussusceptum. In addition, CT may be able to identify the lead point responsible for the intussusception, exclude other causes of abdominal pain or intestinal obstruction, and evaluate for complications such as perforation and bowel ischaemia.

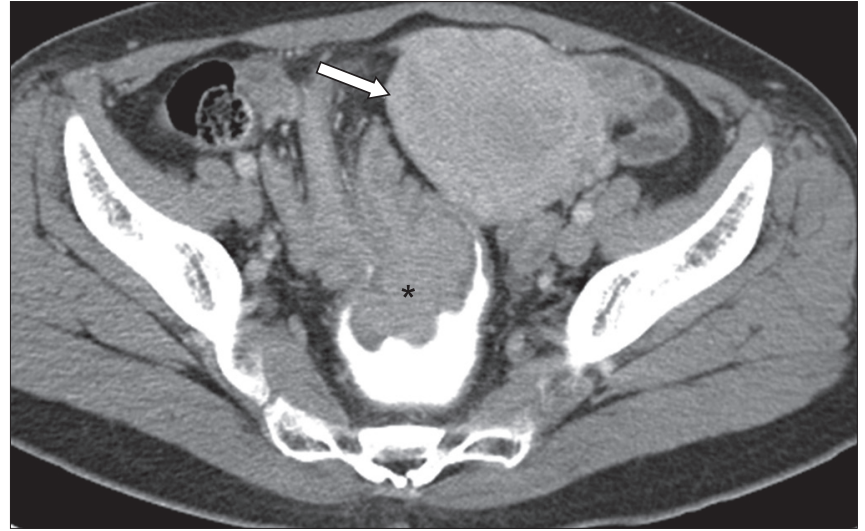

Fig. 4 Contrast-enhanced axial CT image of a female patient shows a colorectal intussusception with a polypoid soft-tissue mass (asterisk) at the apex of the intussusceptum. It is outlined by positive intraluminal contrast media in the rectum. The uterus is indicated by the white arrow.

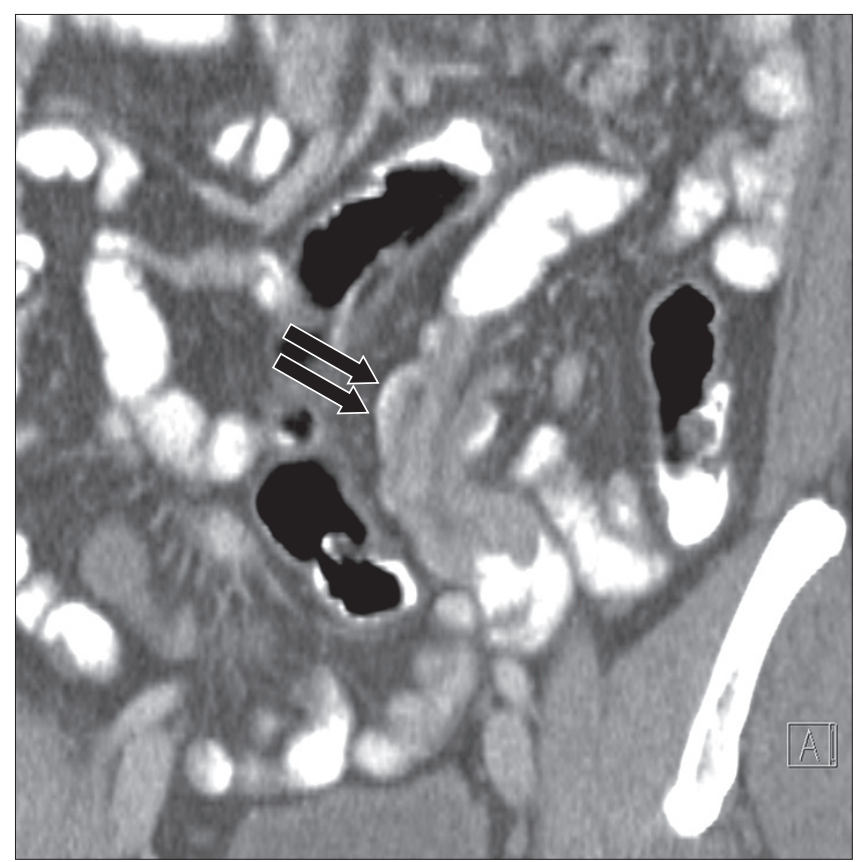

Fig. 5 Contrast-enhanced oblique coronal CT of the abdomen performed in a patient who presented with weight loss and anaemia. A small bowel intussusception (black arrows) is seen in the left lower quadrant with no features of intestinal obstruction. No lead point is identified.

The accuracy of CT in diagnosing Al was shown to be $78 \%$ in one study. ${ }^{(1)}$ In another study, the sensitivity of CT was $>85 \%$ for single- and multi-slice scanners. ${ }^{(11)}$ The drawbacks of CT include the use of ionising radiation in acquiring images and the possibility of breathing artefacts in ill patients.

Ultrasonography is essential in the diagnosis of intussusception in children. The small body habitus of most children allows for the use of high-frequency transducers to produce images with good spatial resolution. The diagnosis relies on the recognition of the 'bowel-within-bowel' appearance on ultrasonography. The 'target' and 'pseudokidney' signs describe the appearance of the intussusception in the transverse and longitudinal planes, respectively. Ultrasonography has a high sensitivity for the diagnosis of intussusception in children $(98 \%-100 \%)^{(12)}$ It is also advantageous, as it does not utilise ionising radiation and allows for portable imaging if the patient 


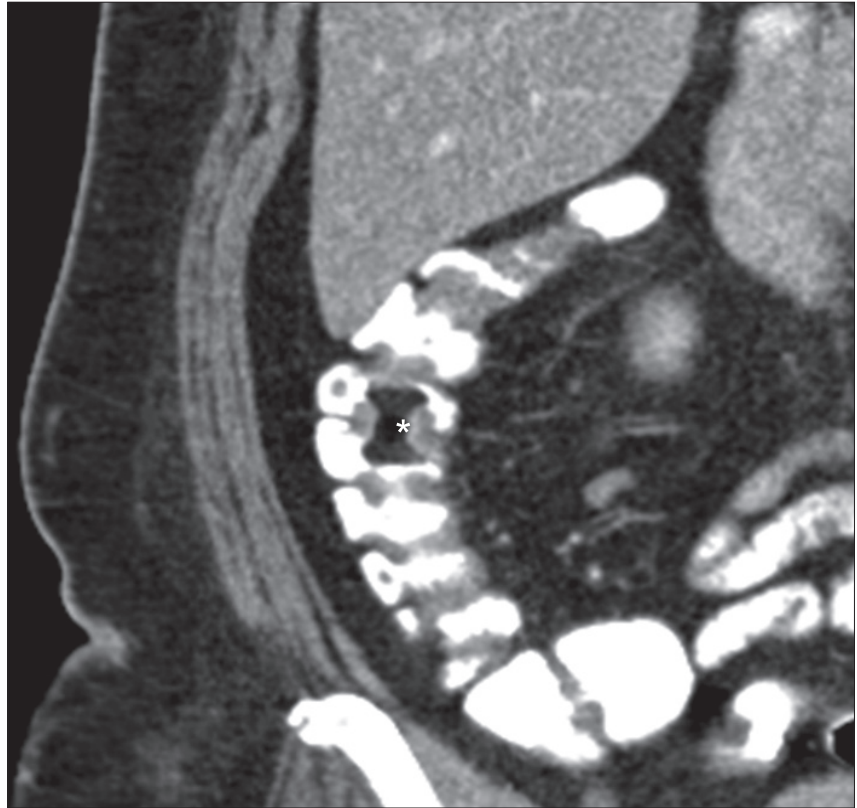

Fig. 6 Contrast-enhanced coronal CT image shows a lipoma (asterisk) within the ascending colon in a patient who was evaluated for an unrelated condition. The lipoma is outlined by positive contrast media within the ascending colon.

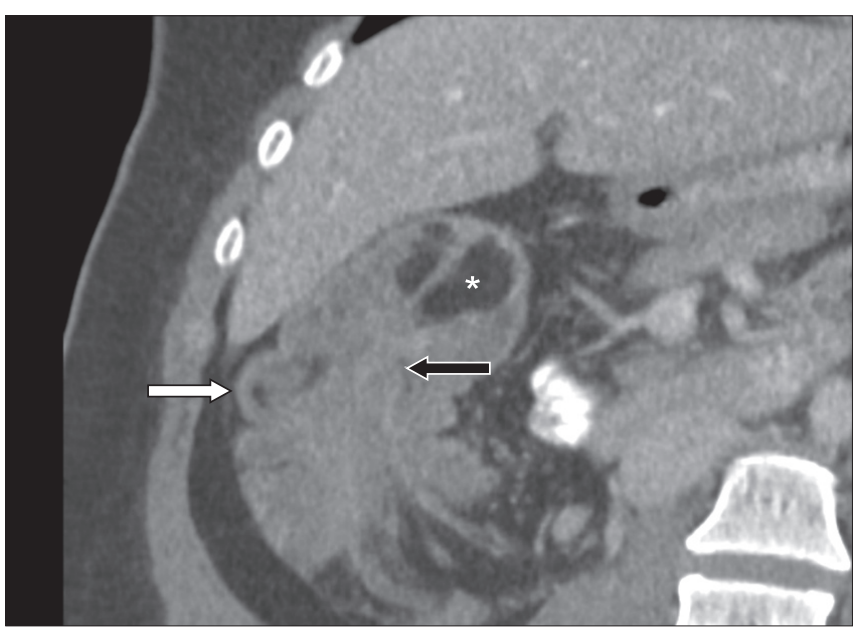

Fig. 7 Coronal CT image of the abdomen and pelvis in portal venous phase shows a lipoma (asterisk) at the apex of the intussusceptum (black arrow). The ascending colon (white arrow) is the intussuscipiens. Laparotomy showed a caecal-colic intussusception.

is too ill to be transported to the radiology department. Despite these advantages, the accuracy of ultrasonography in the diagnosis of $\mathrm{Al}$ is low (50\%). ${ }^{(13)}$ This can be attributed to operator experience, the larger size of adult subjects, and the presence of bowel gas, which leads to poor transmission and difficulty in image interpretation.

Given that a large proportion of Als have a lead point, which incidentally also has a relatively high incidence of malignancy,
ABSTRACT A 50-year-old Chinese man presented with abdominal pain associated with bloody mucoid stools, loss of appetite and weight loss. Contrastenhanced computed tomography of the abdomen and pelvis revealed a colocolic intussusception secondary to a lipoma. The patient subsequently underwent a left hemicolectomy. Clinical and imaging findings of intussusception in adults are discussed in this article.

Keywords: adult intussusception, intestinal obstruction, lipoma, multidetector computed tomography

surgical resection is the recommended treatment, regardless of whether the intussusception is located in the colon or small bowel. ${ }^{(5,14)}$ In contrast, most cases of intussusception in children do not have a lead point. As such, fluoroscopically-guided air-enema reduction is the recommended treatment. Surgical exploration is considered if there are multiple recurrences of intussusception despite successful air-enema reduction, failed air-enema reduction, or when there are signs of perforation, bowel gangrene or necrosis. ${ }^{(12)}$

\section{REFERENCES}

1. Azar T, Berger DL. Adult intussusception. Ann Surg 1997; 226:134-8.

2. Mandeville K, Chien M, Willyerd FA, et al. Intussusception: clinical presentations and imaging characteristics. Pediatr Emerg Care 2012; 28:842-4.

3. Choi SH, Han JK, Kim SH, et al. Intussusception in adults: from stomach to rectum. AJR Am J Roentgenol 2004; 183:691-8.

4. Wang N, Cui XY, Liu Y, et al. Adult intussusception: a retrospective review of 41 cases. World J Gastroenterol 2009; 15:3303-8.

5. Felix EL, Cohen MH, Bernstein AD, Schwartz JH. Adult intussusception; case report of recurrent intussusception and review of the literature. Am J Surg 1976; 131:758-61.

6. Mateen MA, Saleem S, Rao PC, Gangadhar V, Reddy DN. Transient small bowel intussusceptions: ultrasound findings and clinical significance. Abdom Imaging 2006; 31:410-6.

7. Lvoff N, Breiman RS, Coakley FV, Lu Y, Warren RS. Distinguishing features of self-limiting adult small-bowel intussusception identified at CT. Radiology 2003; 227:68-72.

8. Weinberg T, Feldman M Sr. Lipomas of the gastrointestinal tract. Am J Clin Pathol 1955; 25:272-81.

9. Chiang JM, Lin YS. Tumor spectrum of adult intussusception. J Surg Oncol 2008; 98:444-7.

10. Namikawa T, Hokimoto N, Okabayashi T, et al. Adult ileoileal intussusception induced by an ileal lipoma diagnosed preoperatively: report of a case and review of the literature. Surg Today 2012; 42:686-92.

11. Amr MA, Polites SF, Alzghari M, et al. Intussusception in adults and the role of evolving computed tomography technology. Am J Surg 2015; 209:580-3.

12. Chinchure D, Fortier MV. Role of radiology in diagnosis and treatment of an infant with acute abdomen. Ann Acad Med Singapore 2011; 40:154-5.

13. Honjo $\mathrm{H}$, Mike M, Kusanagi $\mathrm{H}$, Kano N. Adult intussusception: a retrospective review. World J Surg 2015; 39:134-8.

14. Barussaud M, Regenet N, Briennon X, et al. Clinical spectrum and surgical approach of adult intussusceptions: a multicentric study. Int J Colorectal Dis 2006; 21:834-9. 


\section{SINGAPORE MEDICAL COUNCIL CATEGORY 3B CME PROGRAMME} (Code SMJ 201612B)

Question 1. Regarding intussusception in adults:

(a) Small bowel intussusception can be transient and self-limiting.

(b) Patients often present with a palpable mass, 'currant jelly' stool and acute abdominal pain.

(c) Computed tomography $(\mathrm{CT})$ is the investigation of choice.

(d) The lead point is often a benign mass.

Question 2. Regarding intussusception in children:

(a) Ultrasonography is the modality of choice, as it uses ionising radiation to acquire images.

(b) Pneumoperitoneum is a potential complication.

(c) A lead point is often found.

(d) Air-enema reduction is performed under fluoroscopy.

Question 3. Regarding imaging of intussusception:

(a) Identifying the 'bowel-within-bowel' appearance of the bowel loops is essential to diagnosis.

(b) The loop of bowel that herniates into the distal bowel loop is called the intussusceptum.

(c) CT does not use ionising radiation to acquire images.

(d) Ultrasonography is not used in adults for the evaluation of intussusception due to the relatively large body habitus.

Question 4. Regarding the management of intussusception:

(a) Surgery is the recommended treatment for a child with intussusception.

(b) Air-enema reduction is the recommended treatment for intussusception in adults.

(c) Surgery is indicated in a patient with intussusception complicated by perforation.

(d) Patients found to have small bowel intussusception on CT, which was done for an unrelated reason, require urgent surgery.

Question 5. Regarding lead points in intussusceptions:

(a) Intussusception in adults is often due to a malignant tumour acting as a lead point.

(b) Most small bowel intussusceptions are due to a malignant tumour.

(c) Lipoma is one of the most common causes of a benign lead point.

(d) The lead point is usually found at the apex of the intussusceptum.

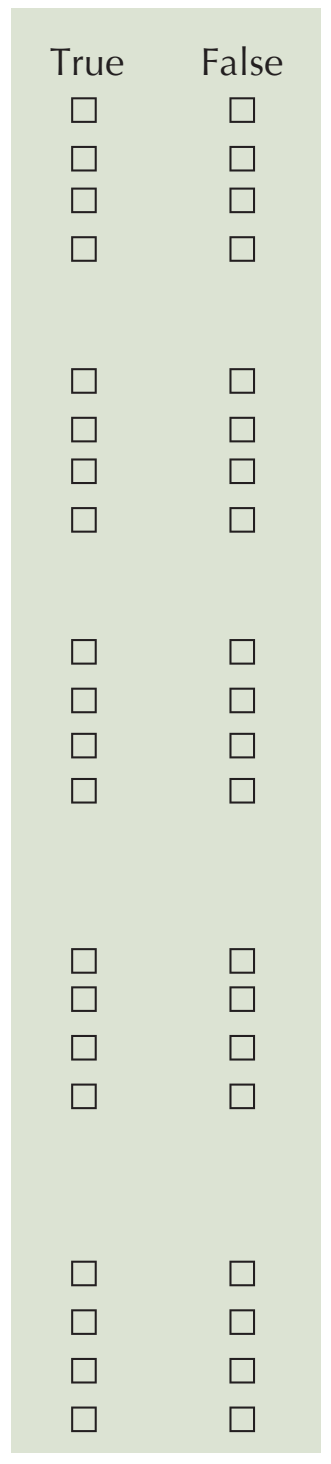

\footnotetext{
Doctor's particulars:

Name in full

MCR number

Email address

Specialty:

SUBMISSION INSTRUCTIONS:

(1) Visit the SMJ website: http://www.smj.org.sg/current-issue and select the appropriate set of questions. (2) Provide your name, email address and MCR number. (3) Select your answers and click "Submit".

RESULTS:

(1) Answers will be published online in the SMJ February 2017 issue. (2) The MCR numbers of successful candidates will be posted online at the SMJ website by 2 February 2017. (3) Passing mark is $60 \%$. No mark will be deducted for incorrect answers. (4) The SMJ editorial office will submit the list of successful candidates to the Singapore Medical Council. (5) One CME point is awarded for successful candidates.

Deadline for submission: (December 2016 SMJ 3B CME programme): 12 noon, 26 January 2017.
} 